\title{
Lightning-driven inner radiation belt energy deposition into the atmosphere: implications for ionisation-levels and neutral chemistry
}

\author{
C. J. Rodger ${ }^{1}$, C.-F. Enell ${ }^{2}$, E. Turunen ${ }^{2}$, M. A. Clilverd ${ }^{3}$, N. R. Thomson ${ }^{1}$, and P. T. Verronen ${ }^{4}$ \\ ${ }^{1}$ Department of Physics, University of Otago, Dunedin, New Zealand \\ ${ }^{2}$ Sodankylä Geophysical Observatory, Sodankylä, Finland \\ ${ }^{3}$ Physical Sciences Division, British Antarctic Survey, Cambridge, UK \\ ${ }^{4}$ Earth Observation, Finnish Meteorological Institute, Helsinki, Finland
}

Received: 14 February 2007 - Revised: 20 July 2007 - Accepted: 31 July 2007 - Published: 29 August 2007

\begin{abstract}
Lightning-generated whistlers lead to coupling between the troposphere, the Van Allen radiation belts and the lower-ionosphere through Whistler-induced electron precipitation (WEP). Lightning produced whistlers interact with cyclotron resonant radiation belt electrons, leading to pitchangle scattering into the bounce loss cone and precipitation into the atmosphere. Here we consider the relative significance of WEP to the lower ionosphere and atmosphere by contrasting WEP produced ionisation rate changes with those from Galactic Cosmic Radiation (GCR) and solar photoionisation. During the day, WEP is never a significant source of ionisation in the lower ionosphere for any location or altitude. At nighttime, GCR is more significant than WEP at altitudes $<68 \mathrm{~km}$ for all locations, above which WEP starts to dominate in North America and Central Europe. Between 75 and $80 \mathrm{~km}$ altitude WEP becomes more significant than GCR for the majority of spatial locations at which WEP deposits energy. The size of the regions in which WEP is the most important nighttime ionisation source peaks at $\sim 80 \mathrm{~km}$, depending on the relative contributions of WEP and nighttime solar Lyman- $\alpha$. We also used the Sodankylä Ion Chemistry (SIC) model to consider the atmospheric consequences of WEP, focusing on a case-study period. Previous studies have also shown that energetic particle precipitation can lead to large-scale changes in the chemical makeup of the neutral atmosphere by enhancing minor chemical species that play a key role in the ozone balance of the middle atmosphere. However, SIC modelling indicates that the neutral atmospheric changes driven by WEP are insignificant due to the short timescale of the WEP bursts. Overall we find that WEP is a significant energy input into some parts of the lower ionosphere, depending on the latitude/longitude and altitude, but does not play a significant role in the neutral chemistry of the mesosphere.
\end{abstract}

Correspondence to: C. J. Rodger

(crodger@physics.otago.ac.nz)
Keywords. Ionosphere (Ion chemistry and composition; Ionization mechanisms; Wave-particle interactions)

\section{Introduction}

Whistler-induced electron precipitation from the Van Allen radiation belts occurs as a result of coupling between the troposphere and the magnetosphere. The energetic electron precipitation arises from lightning produced whistlers (Storey, 1953) interacting with cyclotron resonant radiation belt electrons near the equatorial zone (Tsurutani and Lakhina, 1997). Pitch angle scattering of energetic radiation belt electrons (Kennel and Petschek, 1966) by whistler mode waves drives some resonant electrons into the bounce loss cone, resulting in their precipitation into the atmosphere (Rycroft, 1973). For some electron energy ranges Whistler-induced Electron Precipitation (WEP) is a significant inner radiation belt loss process (e.g. Dungey, 1963; Rodger et al., 2003), acting as one of the drivers by which whistler mode waves (e.g. plasmaspheric hiss, lightning-generated whistlers) cause pitch angle scattering.

One complementary technique to study WEP makes use of long range remote sensing of very low frequency (VLF) waves propagating inside the waveguide bounded by the lower ionosphere and the Earth's surface. Significant variations in the received amplitude and/or phase of fixed frequency VLF transmissions arise from changes in the lower ionosphere. Further discussion on the use of subionospheric VLF propagation as a remote sensing probe can be found in recent review articles (e.g. Barr et al., 2000; Rodger, 2003). WEP leads to localized ionospheric modifications produced by secondary ionisation just below the D-region of the ionosphere, which are observed as "Trimpi" perturbations in subionospheric VLF transmissions (Helliwell et al., 1973). These perturbations begin with a relatively fast $(\sim 1 \mathrm{~s})$ change in the received amplitude and/or phase, followed by

Published by Copernicus Publications on behalf of the European Geosciences Union. 
a slower relaxation $(<100 \mathrm{~s})$ back to the unperturbed signal level due to the recombination of the additional ionisation. Trimpi perturbations permit observers to study WEP fluxes and the chemistry of the nighttime lower ionosphere (e.g. Pasko and Inan, 1994), from locations remote from the actual precipitation region.

Recent studies into the magnitude and time decay of Trimpi perturbations observed at Faraday, Antarctica, have clarified the relationships between WEP mean precipitation energy flux, WEP energy spectra, and VLF perturbation scattering of WEP produced modifications to the ionosphere (Rodger et al., 2004b). Building on this modelling, the dependence of WEP precipitation fluxes on the strength of the associated lightning's return stroke peak current has been identified (Clilverd et al., 2004). In previous studies it has been shown that lightning return stroke current strengths have an approximately linear relationship with the received whistler field strength, and whistler strengths with the size of Trimpi perturbations (Carpenter and LaBelle, 1982; Carpenter and Orville, 1989), from which we would expect a relation between Trimpi scatter amplitude and lightning currents.

Clilverd et al. (2004) considered four study days during which a high proportion of the lightning activity occurring near the east coast of N. America produced observable Trimpi effects on VLF transmitter signals propagating in the region of the Antarctic Peninsula $(\sim L=2-2.5)$. The dependence of the scattered field amplitude on the return stroke peak current of the lightning discharge suggests that during these events diffusion conditions are occurring near the loss cone of the precipitating radiation belt particles due to strong whistler wave fields, probably caused by ducted signals. Three of the four study days showed a high degree of consistency between the levels of lightning return stroke peak current required to produce any given perturbation scatter amplitude value, providing a link between the lightning currents and WEP energy flux (Clilverd et al., 2004). On the remaining day, the Trimpi signatures observed were 6-7 dB greater for a given lightning intensity than on the other study days, consistent with significantly harder radiation belt precipitation spectra (Rodger et al., 2004b), probably caused by post-geomagnetic storm acceleration processes of radiation belt electrons (Meredith et al., 2002).

An important parameter for determining the overall importance of WEP to radiation belt losses is the magnitude of a "typical" WEP event. This may be calculated from theoretical studies (e.g. Abel and Thorne, 1998) or inferred from experimental observations, such as in-situ measurements of WEP events (Voss et al., 1998). A different approach has been to use experimental observations of VLF Trimpi perturbations to characterize typical WEP magnitudes. Investigating the coupling between the troposphere, radiation belts and the middle atmosphere provides information about radiation belt losses, while at the same time leading to new understanding on energy inputs into the middle atmosphere/lower ionosphere. In our previous studies, we considered the lifetimes of radiation belt electrons due to WEP losses, based on Trimpi measurements and an estimate of the a typical WEP burst mean precipitation energy flux (e.g. Rodger and Clilverd, 2002). The reliability of those rates and the validity of the conclusions drawn from those studies was tested by examining a new set of Trimpi observations from New Zealand (Rodger et al., 2005). About $10000 \mathrm{~h}$ of subionospheric very-low frequency (VLF) recordings made from 17 April 2003 through to 26 June 2004 in New Zealand were examined, searching for Trimpi perturbations observed on transmissions from VLF transmitters in Hawaii (NPM) and western Australia (NWC). The occurrence rates from New Zealand were compared with those previously reported from the Antarctic peninsula. The perturbation rates observed in the New Zealand data were found to be consistent with those predicted from the global distribution of the lightning sources, allowing for the different experimental configurations. This provides confidence in the global WEP rate map presented in the 2005 study and also the basic conclusions of our earlier studies (e.g. Rodger et al., 2003, 2004a). Using lightning current distributions rather than VLF perturbation observations Rodger et al. (2005) revised their previous estimates of typical precipitation bursts at $L \sim 2.3$ to a mean precipitation energy flux of $\sim 1 \times 10^{-3} \mathrm{ergs} \mathrm{cm}^{-2} \mathrm{~s}^{-1}$. This study went on to consider how the energy flux deposited into the atmosphere by WEP bursts will behave globally, estimating the regional variation in precipitation as an energy input to the middle-atmosphere. The precipitation of energetic electrons by these bursts in the range $L=1.9-3.5$ means that the mean rate of energy deposited into the atmosphere by WEP is $5 \times 10^{-6} \mathrm{ergs} \mathrm{cm}^{-2} \mathrm{~s}^{-1}$, spatially varying from a low of zero above some ocean regions to highs of $\sim 5-$ $10 \times 10^{-5} \mathrm{ergs} \mathrm{cm}^{-2} \mathrm{~s}^{-1}$ above North America and its conjugate region.

Satellite observations indicate that the global annual average total lightning flash rate is $44 \pm 5$ flashes per second (Christian et al., 2003), while Clilverd et al. (2004) found that the most significant WEP bursts were due to lightning with return stroke peak currents of $I \geq 70 \mathrm{kA}$, which includes $\sim 14 \%$ of all cloud to ground lightning. As such the global annual average rate for significant WEP bursts should be $\sim 1.5 \mathrm{~s}^{-1}$, with all remaining lightning flashes producing a much higher rate of WEP bursts with smaller intensities. In general, it is understood that there are $\sim 3.5$ times more intracloud (IC) lightning strokes (sometimes termed "cloud discharges") lightning flashes than CG flashes (Mackerras et al., 1998), although CG discharges are "stronger", with higher peak currents. As previous studies have described how the WEP energy spectra varies with geomagnetic latitude, and the WEP flux magnitude varies with latitude and longitude, we are now in a position to describe the variation in WEPdriven ionisation rates with altitude over the entire Earth. In this paper we examine the significance of WEP as an ionisation source in the lower ionosphere, by contrasting the 


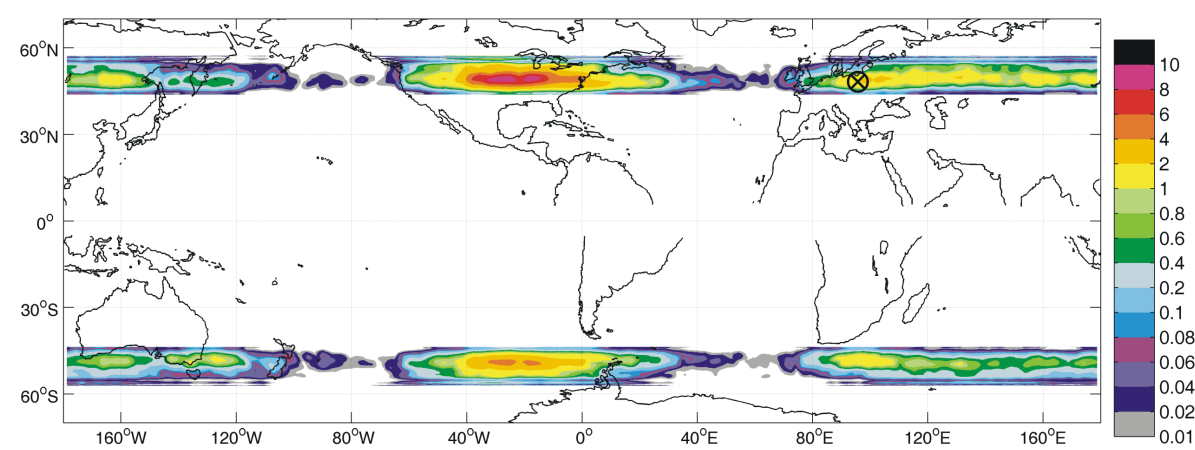

Fig. 1. Map showing the expected global distribution of energy flux deposited by WEP into the atmosphere in CGM coordinates, in units of $10^{-5} \mathrm{ergs} \mathrm{cm}^{-2} \mathrm{~s}^{-1}$ (after Rodger et al., 2005).

importance of WEP-driven ionisation rates with those from the other primary sources, galactic cosmic radiation and solar photoionisation. Previous studies have also shown that particle precipitation (e.g. solar proton events) can lead to largescale changes in the chemical makeup of the neutral atmosphere as increases in ionisation rates enhance minor chemical species that play a key role in the middle atmosphere (e.g. ozone). Thus we also investigate the significance of WEP to the chemical makeup of the mesosphere.

\section{Variation in WEP-produced ionisation rates}

\subsection{Global distribution of WEP energy deposition}

Rodger et al. (2005) provided an estimate of the global variation in mean precipitation energy flux due to WEP deposited into the atmosphere by combining Trimpi observations, the average geographical distribution of total lightning activity from Optical Transient Detector satellite observations (Christian et al., 2003), and in-situ S81-1 satellite observations of the $L$-shell and time signature of WEP (Voss et al., 1998). The modelling undertaken in that study assumes an electron beam limited to the range $1-1500 \mathrm{keV}$, produced by a ducted whistler spanning $0.5-5 \mathrm{kHz}$ with power spectral density as given by the lightning spectrum. These authors assumed that the WEP burst lasts $0.2 \mathrm{~s}$, and used a cold plasma density taken from Menk et al. (1999). As the starting point of the current study we show a slightly modified version of their map of mean precipitation energy flux due to WEP deposited into the atmosphere as Fig. 1, in this case with units of $10^{-5} \mathrm{ergs} \mathrm{cm}^{-2} \mathrm{~s}^{-1}$. This plot presents the variation in energy precipitated by global WEP activity in Corrected GeoMagnetic (CGM) coordinates based on the Definite/International Geomagnetic Reference Field (DGRF/IGRF) for 2003 at $100 \mathrm{~km}$ altitude, using the GEOPACK software routines. To aid the eye, this figure shows the geographical coastlines also translated into CGM coordinates.
The strong latitudinal banding in Fig. 1 is a consequence of the coupling between lightning and the radiation belts, which varies with $L$. In situ observations by the S81-1 satellite indicate that WEP is rare at low $L$-shells (Voss et al., 1998), despite high lightning activity near the equator, due to increasingly unfavourable gyroresonance conditions (Friedel and Hughes, 1992). This coupling $L$-variation was estimated from S81-1/SEEP measurements of how experimentally observed WEP varies with $L$ (Fig. 11, Voss et al., 1998). The plot includes both "primary" WEP (from lightning in the same hemisphere) and "secondary" WEP (from conjugate lightning), producing high WEP rates in both hemispheres at American longitudes due to North American lightning. However, there is no symmetry between the Northern and Southern Hemispheres in Fig. 1 as more electrons precipitate into the lightning-source hemisphere than into the conjugate hemisphere. In addition, lightning activity is more common in the Northern Hemisphere where there is more land, such that the rate of energy deposited by WEP into the atmosphere is higher for the Northern Hemisphere than the Southern. Our calculation assumes ducted propagation and cyclotron resonance with counter-streaming energetic electrons through the $m=1$ resonance mode. Calculations including oblique whistler propagation (e.g. Bortnik et al., 2006) would have harder spectra at higher latitudes, and hence more ionisation at lower altitudes.

Figure 1 provides the WEP mean precipitation energy flux, necessary to determine the overall significance of WEP as an ionospheric D-region ionisation source at different locations round the world.

\subsection{Global variation in WEP-driven ionisation rate}

From the mean precipitation energy flux we calculate the WEP-produced mean ionisation rate, to allow comparison with other sources of ionisation in the D-region of the ionosphere. The spatially varying mean WEP energy fluxes shown in Fig. 1 are combined with WEP energy spectra, in both cases calculated following the approach outlined in Rodger et al. (2003, 2004b). The parameters of the WEP 
burst were selected by comparison with the calculations of Faraday Trimpi produced by WEP with a mean precipitation energy flux at $L=2.23$ of $2 \times 10^{-3} \mathrm{ergs} \mathrm{cm}^{-2} \mathrm{~s}^{-1}$ (Rodger et al., 2004b), specifically the "normal" Faraday Trimpi spectral conditions (Clilverd et al., 2004). Following the approach outlined in the Rodger et al. (2003, 2004b, 2005) studies, this starting point is used to derive global variations. The typical WEP rates necessary to determine the Fig. 1 WEP energy fluxes were described in Rodger et al. (2005).

The WEP energy spectrum is determined from the spectrum of the trapped electrons in the pitch angle range from the loss cone angle $\left(\alpha_{L C}\right)$ to $\left(\alpha_{L C}+0.5^{\circ}\right)$ given by combining the differential omnidirectional electron fluxes of the ESA-SEE1 electron radiation belt model with the CRRESsatellite observed pitch angle dependences (Vampola, 1996) for $3<L<6.75$ and those from the earlier empirical AE-5 radiation belt model (Teague and Vette, 1972). A detailed description of the ESA-SEE1 model and its combination with AE-5 was described in Rodger et al. (2006). The resulting electron radiation belt model is used to provide typical "quiet-time" WEP energy spectra. Intense geomagnetic disturbances can inject energetic electrons into the slot and inner radiation belt, leading to a harder energy spectrum for the trapped population. In these cases the WEP energy spectra will also harden, as has been experimentally observed (Inan et al., 1989; Clilverd et al., 2004). As injections into the inner radiation belt are quite rare, we are able to assume that the "quiet" WEP energy spectra are more indicative of typical conditions. This WEP flux and spectra, representing the mean energy deposited by WEP into a given location into the atmosphere, is finally used to create WEP-produced ionisation rates by an application of the expressions in Rees (1989). The background neutral atmosphere is calculated using the NRLMSISE-00 neutral atmospheric model (Picone et al., 2002).

Figure 2 shows the globally varying ionisation rate due to the mean WEP energy fluxes shown in Fig. 1, again in CGM coordinates. Ionisation rates are shown with units $\log _{10}\left(\right.$ electrons $\left.\mathrm{cm}^{-3} \mathrm{~s}^{-1}\right)$. Satellite observations of WEP indicate there is essentially no activity outside of $L=1.9-3.5$, leading to bands of magnetic latitudes in which WEP ionisation rates may be non-zero. Only locations with non-zero WEP energy fluxes are shown on the plot, a format we will continue through the paper to allow easy comparisons. While calculations were undertaken from $40-90 \mathrm{~km}$ altitudes, only the altitudes spanning 55-90 km are shown in $5 \mathrm{~km}$ steps. All eight panels have the same colour scale, stretching over the ionisation rate range from $10^{-3.5}$ to $10^{0.5}$ electrons $\mathrm{cm}^{-3} \mathrm{~s}^{-1}$. Below $55 \mathrm{~km}$ there is essentially no WEP-driven ionisation produced as the energy spectra are too soft, and significant penetration does not occur to these altitudes. Below $60 \mathrm{~km}$ the mean WEP-driven ionisation rate is less than $2 \times 10^{-4}$ electrons $\mathrm{cm}^{-3} \mathrm{~s}^{-1}$ for all spatial locations considered. This ionisation rate is very small, and is not significant when contrasted with other ionisation sources at these alti- tudes (and below), as will be shown below. As expected for WEP bursts, which typically involve electrons of energies from tens to hundreds of $\mathrm{keV}$ (Rodger et al., 2003, Fig. 2), depending on $L$-shell, the peak ionisation rates at larger $L$ shells are at higher altitudes, where high-fluxes of low energy electrons will be deposited.

\section{Comparison with other ionisation sources}

\subsection{Global variation in GCR-driven ionisation rate}

Galactic Cosmic Radiation (GCR) is the primary source of ionisation in the stratosphere, and is also a significant ionisation-driver for the lower mesosphere, particularly at night. The ionisation rate due to GCR has been determined at a given geomagnetic latitude and longitude following Heaps (1978).

Figure 3 shows a comparison between the mean ionisation rate due to WEP (black lines) with that caused by GCR (green lines), for the altitude range $55-90 \mathrm{~km}$ and various longitudes. The lines marked with circles show the ionisation rates for the Southern Hemisphere, while the solid lines show those for the Northern Hemisphere. In some cases in Fig. 3, the lines with circles appear simply as a thicker coloured line. The ionisation rates shown are means for locations across the Northern and Southern Hemisphere latitudes with non-zero WEP energy fluxes, for each longitude value. As noted earlier, at the lowest altitudes GCR is always more important than WEP everywhere across the planet. As the altitude increases, WEP first becomes dominant above North America and Central Europe at $68 \mathrm{~km}$. Between 75 and $80 \mathrm{~km}$ altitude WEP becomes more significant than GCR for the majority of spatial locations at which WEP deposits energy.

\subsection{Global variation in daytime solar Lyman- $\alpha$}

For most of the mesosphere, the dominant source of ionisation production during the day is due to photoionisation of the neutral atmosphere by electromagnetic radiation from the Sun. The dominant mechanism during the day is the ionisation of nitric oxide (NO) by solar Lyman- $\alpha(121.6 \mathrm{~nm})$, and to a lesser extent EUV (102.7-111.8 nm) acting on $\mathrm{O}_{2}\left({ }^{1} \Delta_{g}\right)$. In order to determine the significance of WEP relative to solar photoionisation, we calculate the solar photoionisation ionisation rate at each non-zero WEP energy flux location using the highest solar zenith angle possible for that point on the Earth. The solar flux is calculated from $0.5-417.5 \mathrm{~nm}$ with the spectrum and cross-sections used in the pure ion-chemistry version of the SIC model (Turunen et al., 1996) providing a photoionisation source incident upon a NRLMSISE-00 neutral atmosphere containing 8 constituents: $\mathrm{N}_{2}, \mathrm{O}_{2}, \mathrm{O}, \mathrm{O}_{3}, \mathrm{NO}, \mathrm{CO}_{2}, \mathrm{H}_{2} \mathrm{O}, \mathrm{O}_{2}\left({ }^{1} \Delta_{g}\right)$.

Our calculations shown that when contrasted with mean WEP produced ionisation rates, daytime solar photoionisation dominates for all locations above the altitude of $63 \mathrm{~km}$. 

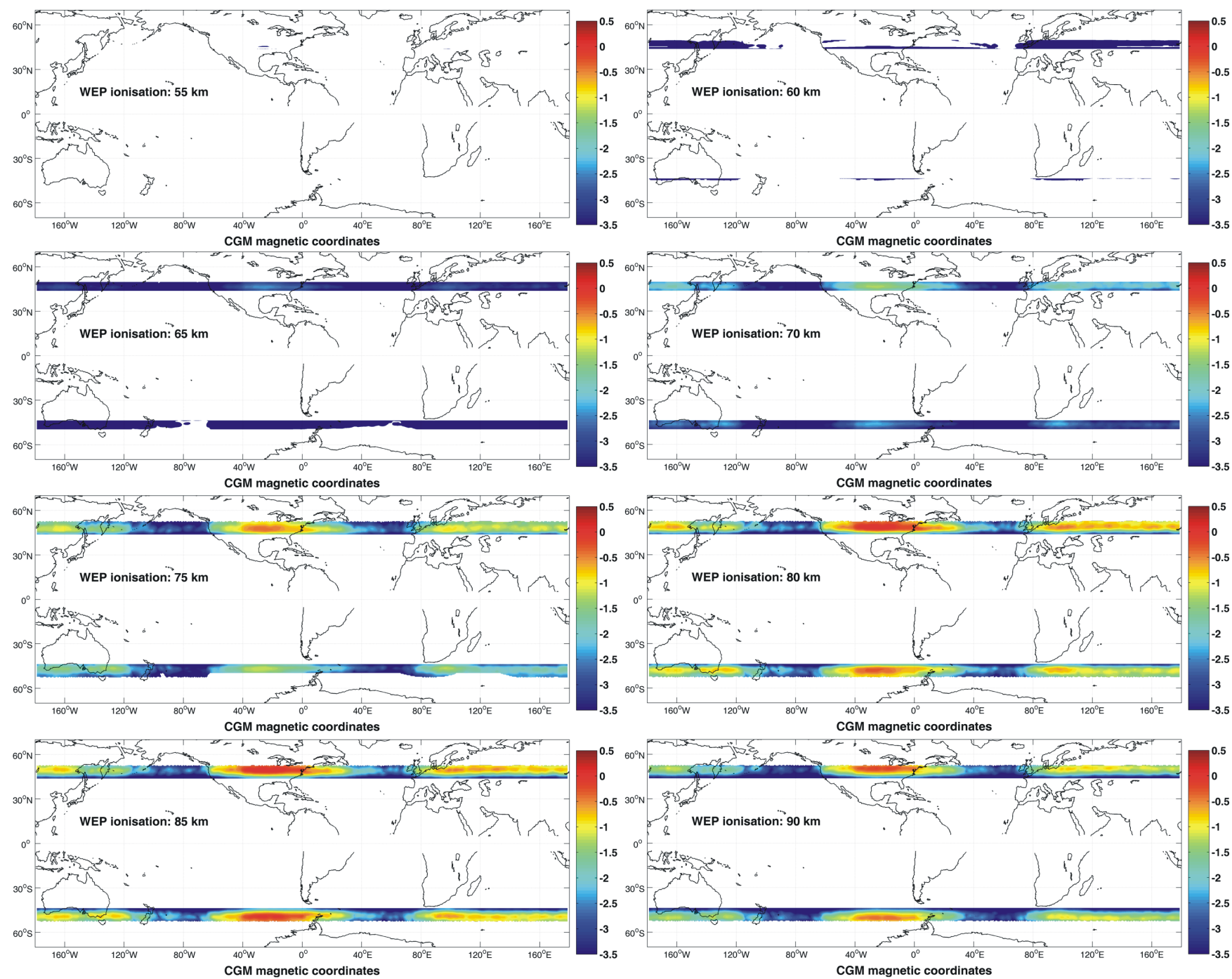

Fig. 2. Maps showing the mean ionisation rate due to WEP into the atmosphere, based on the energy deposition shown in Fig. 1. Ionisation rates are shown with units $\log _{10}$ (electrons $\mathrm{cm}^{-3} \mathrm{~s}^{-1}$ ). Only those locations and altitudes with non-zero energy fluxes are included in the plotting.

While WEP drives a higher ionisation rate than daytime solar photoionisation in some parts of the Earth at lower altitudes, at those altitudes both the photoionisation and WEP ionisation rates are many orders of magnitude smaller than the ionisation rate due to Galactic Cosmic Radiation (Fig. 3). Thus WEP is never a significant source of ionisation in the lower ionosphere during the day.

\subsection{Global variation in nighttime solar Lyman- $\alpha$}

The diurnal variation in the solar Lyman- $\alpha(121.6 \mathrm{~nm})$ is naturally substantial. During nighttime there are significantly reduced but non-zero solar Lyman- $\alpha$ fluxes, with scattering from the geocorona leading to reductions by a factor of about one hundred relative to the fluxes at noon (Kazil et al., 2003). The scattered component of solar Lyman- $\alpha$ flux is included in our calculations using the empirical approximation given by Thomas and Bowman $(1985,1986)$ to determine the solar photoionisation rate for local midnight at each non-zero WEP energy flux location.

As seen in Fig. 3, there is a complex relationship between the ionisation rate driven by WEP and that due to nighttime solar Lyman- $\alpha$. Between $75-80 \mathrm{~km}$ altitude WEP is dominant for more than half of the longitude range in the Northern Hemisphere, and in a somewhat smaller longitudinal range in the Southern. Below $60 \mathrm{~km}$ WEP is more important than nighttime solar Lyman- $\alpha$ in the Northern Hemisphere, but not in the Southern. However, in these low altitudes, both WEP and solar Lyman- $\alpha$ are less important than GCR. The transition altitude for which nighttime solar Lyman- $\alpha$ is more important than GCR is $\sim 70 \mathrm{~km}$, above which the primary 

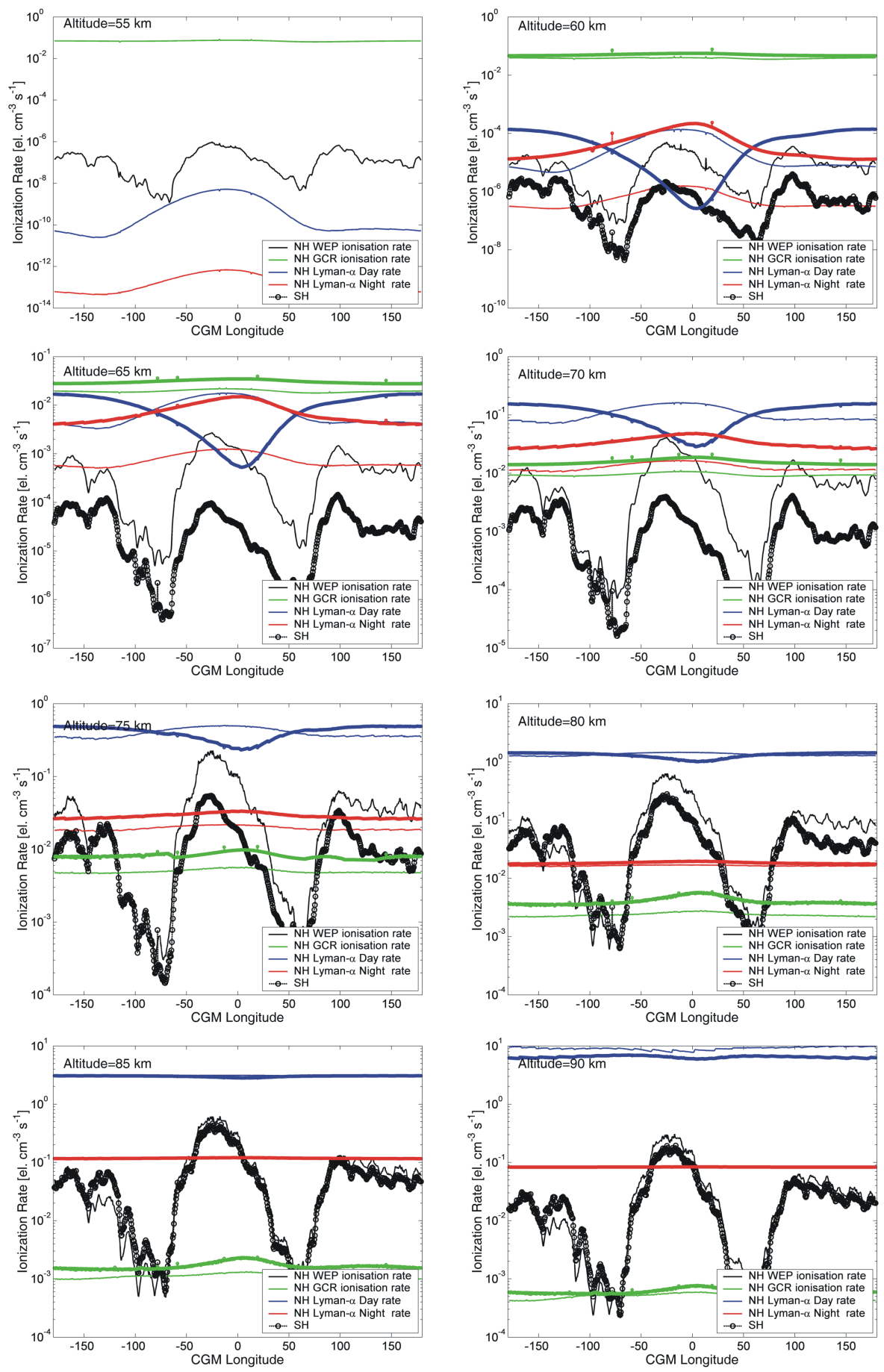

Fig. 3. Contrast between the longitudinally varying latitudinally-averaged ionisation rates for WEP (black line), GCR (green), and midday and midnight photoionisation (blue and red, respectively). Northern Hemisphere values are shown by solid lines, and Southern Hemisphere by lines with circles.

nighttime energy input will be either WEP or solar Lyman$\alpha$. The size of the regions in which WEP is the most important nighttime ionisation source is largest at $\sim 80 \mathrm{~km}$, corresponding to those locations in Fig. 2 with WEP driven ionisation rates of $>3 \times 10^{-2} \mathrm{el} . \mathrm{cm}^{-3} \mathrm{~s}^{-1}$. At all other locations in Fig. 2, nighttime solar Lyman- $\alpha$ remains dominant. For altitudes above $80 \mathrm{~km}$ altitude the spatial region in which WEP is dominant becomes progressively smaller; 
at $90 \mathrm{~km}$ only the zones with WEP driven ionisation rates of $>1 \times 10^{-1}$ el. $\mathrm{cm}^{-3} \mathrm{~s}^{-1}$ overcome nighttime solar Lyman- $\alpha$ as the main ionisation source.

\subsection{Significance of WEP as an ionisation source}

As shown in the previous parts of this section, WEP dominates over both solar photoionisation and GCR over some altitudes at some locations. As an example of the relative importance of the various processes, we contrast the altitude dependent ionisation rates due to all the processes considered for a location with high WEP driven ionisation. This is shown in Fig. 4, where the calculations have been undertaken for the CGM coordinate location of $48.25^{\circ} \mathrm{N}, 95.75^{\circ} \mathrm{E}$ (Poland), marked by a ringed cross in Fig. 1, a location which has a fairly high WEP driven ionisation rate while still being an order of magnitude smaller than the peaks seen in Fig. 2. Figure 4 contrasts the ionisation rate due to WEP (solid line), GCR (circles), daytime and nighttime solar photoionisation (crosses and a dashed line, respectively). During the day and for all altitudes above $66 \mathrm{~km}$, solar photoionisation is the dominant driver for ionisation, while at lower altitudes GCR is the most significant source. However, during the nighttime, the transition height between solar photoionisation, GCR and also WEP is $\sim 69-70 \mathrm{~km}$. Below this GCR is again the most significant source, while WEP is the most important driver of nighttime ionisation at this location for higher altitudes up to $\sim 87 \mathrm{~km}$.

\section{Significance to the atmospheric chemistry}

In the sections above we considered the significance of WEP as an ionisation source in the mesosphere. Previous studies have shown that various particle precipitation mechanisms (e.g. solar proton events) can lead to large-scale changes in the chemical makeup of the neutral atmosphere. Increased ionisation and dissociation rates due to particle precipitation result in enhancements of odd nitrogen $\left(\mathrm{NO}_{\mathrm{x}}\right)$ and odd hydrogen $\left(\mathrm{HO}_{\mathrm{x}}\right) . \mathrm{NO}_{\mathrm{x}}$ and $\mathrm{HO}_{\mathrm{x}}$ play a key role in the ozone balance of the middle atmosphere because they destroy odd oxygen through catalytic reactions (e.g. Brasseur and Solomon, 1986, pp. 291-299). Ionisation and dissociation rate increases in the upper atmosphere caused by solar proton events are known to lead to local perturbations in ozone levels (Verronen et al., 2005). Changes in $\mathrm{NO}_{2}$ and $\mathrm{O}_{3}$ consistent with solar proton-driven modifications have been experimentally observed (Seppälä et al., 2004, 2006; Verronen et al., 2005). Neutral atmospheric chemistry changes are not limited to proton precipitation; Rodger et al. (2006) showed that intense energetic precipitation of radiation belt electrons caused by systems envisaged to control the Van Allen belts (Inan et al., 2003) and protect Earth-orbiting satellites would lead to similarly large changes in odd nitrogen, odd hydrogen and ozone.

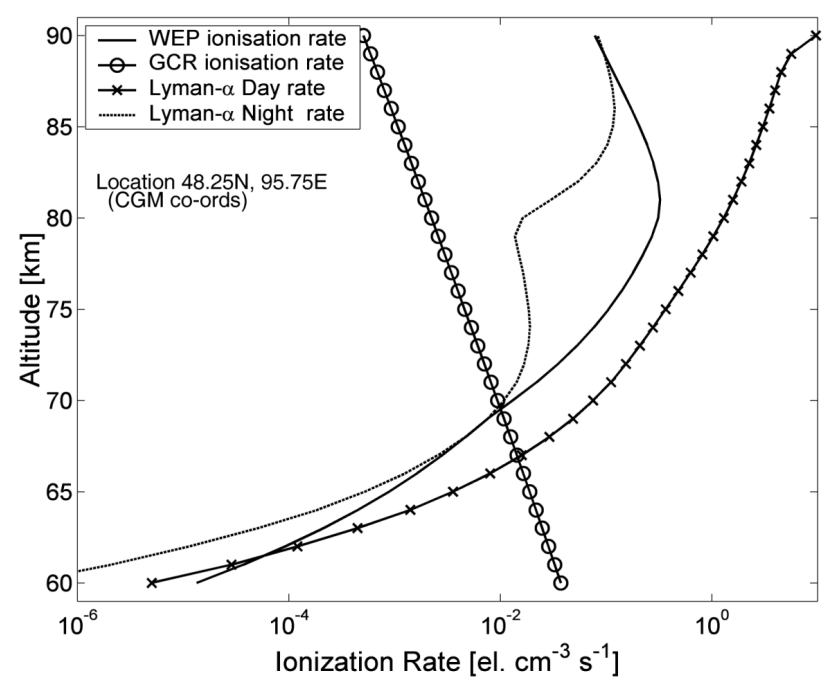

Fig. 4. Contrast between ionisation rates due to differing drivers at the location marked in Fig. 1 (in Poland). The solid line shows the ionisation rate profile with altitude produced by WEP, to be contrasted with that produced by GCR (circles), and midday and midnight photoionisation (crosses and dotted, respectively).

However, the precipitation fluxes from human control of the radiation belts and solar proton events are large and relatively long-lived when contrasted with WEP. As such care needs to be taken before suggesting that WEP-driven ionisation increases could lead to significant changes in neutral atmospheric chemistry. We therefore apply a sophisticated atmospheric chemistry model to consider the impact of a period of high WEP activity.

\subsection{Trimpi observations of WEP bursts}

We make use of observations of Trimpi perturbations recorded at Faraday, Antarctica $\left(65.25^{\circ} \mathrm{S}, 64.27^{\circ} \mathrm{W}\right.$, $L=2.45$ ) on the night of 14 April 1994 from 03:15-11:22 UT. This day was chosen due to a high, but not atypical Trimpi rate. While significant WEP-rates are likely to have occurred outside this time window, higher noise levels degraded Trimpi observation at other times on this day. The scatter amplitude of the Trimpi observed on the night of 14 April 1994 are most consistent with disturbed radiation belt conditions, probably associated with recent geomagnetic disturbances. We take the observation of Trimpi perturbations as a proxy for WEP bursts, noting that previous studies have shown that the Trimpi detection limit at Faraday during disturbed radiation belt conditions occurs at scatter amplitude perturbations of $-35 \mathrm{~dB}$, themselves caused by a $46 \mathrm{kA}$ cloud to ground lightning discharge that leads to a $1.4 \times 10^{-4} \mathrm{ergs} \mathrm{cm}^{-2} \mathrm{~s}^{-1}$ WEP burst (Clilverd et al., 2004). This detection limit suggests that many more WEP bursts should be occurring than given by the observed Trimpi rates, as the majority of WEP bursts will be caused 

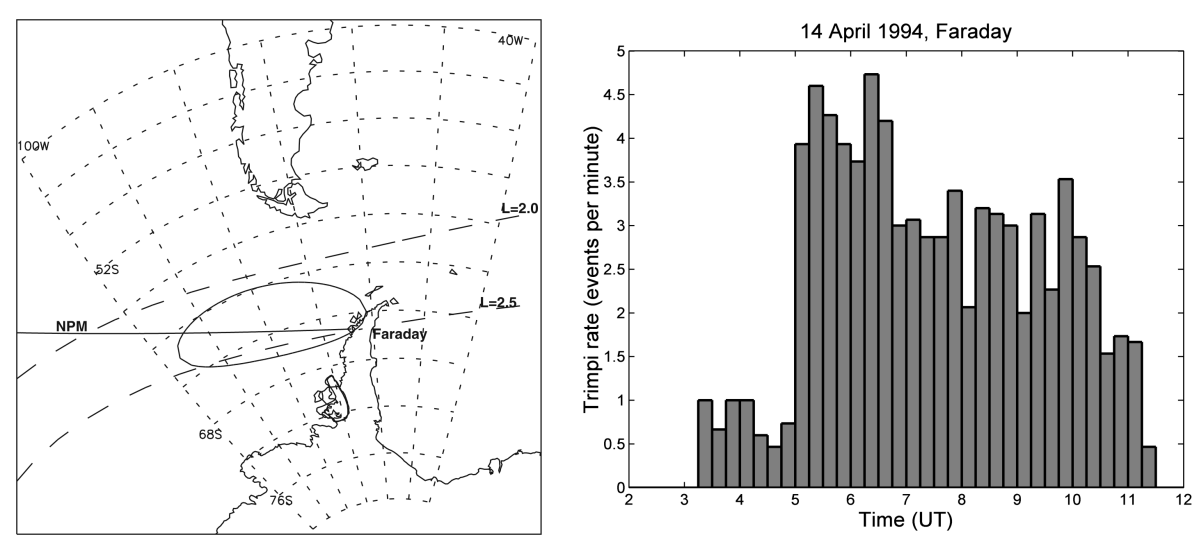

Fig. 5. The left panel shows the region around the Antarctic Peninsula and the location of Faraday Station. The great circle path to NPM is shown, as is the expected location of the WEP produced ionospheric modification. The right panel shows Trimpi rate observations on transmissions from NPM received at Faraday.

by lightning with $I<70 \mathrm{kA}$ and hence will not be detected through subionospheric observations, even though the bursts will be precipitating energetic electrons into the D-region.

Subionospheric signals from the VLF transmitter NPM $(23.4 \mathrm{kHz}$, Oahu, Hawaii) were recorded on an OMSK receiver (Dowden et al., 1994). The analysis of this time period has been previously described by Rodger et al. (2002). Figure 5 shows location of Faraday and WEP-produced ionospheric modifications in the left-hand panel. The right hand panel of this figure shows the Trimpi rate per minute using $15 \mathrm{~min}$ averaging bins. During the $8 \mathrm{~h}$ period 1248 Trimpi were observed, about $85 \%$ of which occurred from 05:0010:30 UT when local noise levels were at their lowest. As such we have a period of $\sim 5.5 \mathrm{~h}$ during which the average Trimpi rate observed at Faraday was $\sim 3.3$ events per minute. An analysis of 115 days from 1993 and 1994 found that at the typical times of highest Trimpi rates the observed rate was higher than $3 / \mathrm{min}$ on only $\sim 10 \%$ of days examined (Rodger et al., 2002), indicating that the conditions on the night of 14 April 1994 were rare, but not extreme.

For WEP events which could be associated with the causative cloud to ground lightning discharge observed by the U.S. National Lightning Detection Network (NLDN) (Cummins et al., 1998), the WEP energy flux is determined by the experimentally observed relationship between the return stroke peak current of the lightning discharge, the Trimpi scatter amplitude perturbation, and the modelled WEP energy flux (Clilverd et al., 2002). For the remaining $80 \%$ of Trimpi events, a random peak current is assumed instead of the NLDN-measured current. The randomly determined current is specified using the empirical CG peak current probability distribution of Popolansky (1972), taking into account that the current value must be greater than $46 \mathrm{kA}$ to produce an observable Trimpi. This provides a description of WEP bursts such that we can determine the impact of this precipitation upon the atmosphere.

\subsection{Sodankylä Ion Chemistry model}

We use the Sodankylä Ion Chemistry (SIC) model to consider the atmospheric consequences of relatively intense WEP activity, through changes in $\mathrm{HO}_{\mathrm{x}}$ and $\mathrm{NO}_{\mathrm{x}}$. The Sodankylä Ion Chemistry (SIC) model is a 1-D chemical model designed for ionospheric D-region studies, solving the concentrations of 64 ions, including 28 negative ions, and 15 neutral species at altitudes in the range $20-150 \mathrm{~km}$. Our study made use of SIC version 6.8.1. The model has recently been discussed by Verronen et al. (2005), building on original work by Turunen et al. (1996) and Verronen et al. (2002). A detailed overview of the model was given in Verronen et al. (2005), but we summarize here to provide background for this study.

In the SIC model over 300 reactions are implemented, plus additional external forcing due to solar radiation (1$422.5 \mathrm{~nm}$ ), electron and proton precipitation, and galactic cosmic radiation. Initial descriptions of the model are provided by Turunen et al. (1996), with neutral species modifications described by Verronen et al. (2002). Solar flux is calculated with the SOLAR2000 model (version 2.21) (Tobiska et al., 2000). The scattered component of solar Lyman$\alpha$ flux is included using the empirical approximation given by Thomas and Bowman (1986). The SIC code includes vertical transport (Chabrillat et al., 2002) which takes into account molecular diffusion coefficients (Banks and Kockarts, 1973). The background neutral atmosphere is calculated using the MSISE-90 model (Hedin, 1991) and tables given by Shimazaki (1984). Transport and chemistry are advanced in intervals of 5 or $15 \mathrm{~min}$, while within each interval exponentially increasing time steps are used because of the wide range of chemical time constants of the modelled species.

\subsection{SIC modelling of WEP forcing}

In the SIC model runs the ionisation and dissociation rates are calculated from the estimated electron energy spectra as 

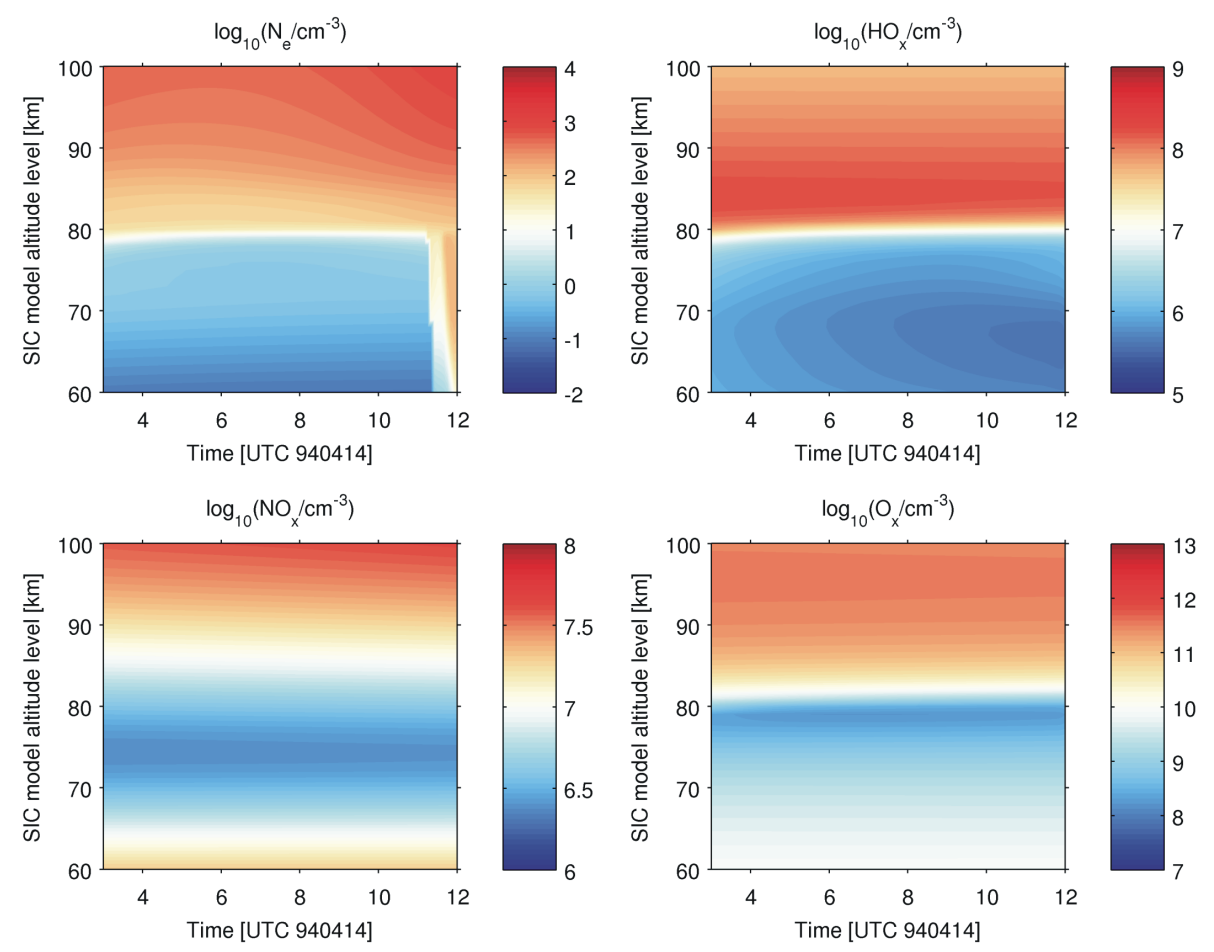

Fig. 6. The results of a SIC modelling run without any WEP-forcing (i.e. zero precipitating electron fluxes), showing the calculated "normal" conditions.

outlined by Turunen et al. (1996) (based on the method of Rees, 1989). Hence we examine the altitude and time variation in neutral atmospheric species, as well as the electron density profile. The SIC model was run for 14 April and at the location of $\left(62.5^{\circ} \mathrm{S}, 280^{\circ} \mathrm{E}, L=2.23\right)$, roughly the centre of the WEP-disturbed ionospheric region shown in Fig. 5. Each WEP burst is assumed to last $0.2 \mathrm{~s}$, following earlier studies. In order to interpret the WEP-driven changes, a SIC modelling run has also been undertaken without any WEP-forcing (i.e. zero precipitating electron fluxes). The results of this no-forcing "control" SIC-run, shown in Fig. 6, allow the calculation of "normal" conditions, and hence an indication of the significance of the changes. The top-left panel of Fig. 6 shows the normal diurnal variation in electron number density, the bottom-left panel shows $\mathrm{NO}_{\mathrm{x}}$ number density $\left(\mathrm{NO}+\mathrm{NO}_{2}\right)$, the top-right hand panel shows $\mathrm{HO}_{\mathrm{x}}\left(\mathrm{OH}+\mathrm{HO}_{2}\right)$, and the bottom-right lower panel shows $\mathrm{O}_{\mathrm{x}}\left(\mathrm{O}+\mathrm{O}_{3}\right)$. We use $\mathrm{NO}_{\mathrm{x}}, \mathrm{HO}_{\mathrm{x}}$ and $\mathrm{O}_{\mathrm{x}}$ rather than $\mathrm{NO}$, $\mathrm{OH}$ and $\mathrm{O}_{3}$ as there are substantial diurnal variations in both the latter populations, which would lead to distracting features in the relative change plots. In all cases these panels have units of $\log _{10}\left[\mathrm{~cm}^{-3}\right]$. The atmospheric changes modelled in our study occur in the mesosphere, as determined by energy spectra of the precipitating electrons. The rapid increase in electron number density from $\sim 11.5$ UT is due to sunrise. This is not seen in the $\mathrm{NO}_{\mathrm{x}}, \mathrm{HO}_{\mathrm{x}}$ and $\mathrm{O}_{\mathrm{x}}$ number densities because although photochemistry changes the equi- librium between the subspecies, the total concentration stays almost constant.

The top panel of Fig. 7 shows the effect of the WEPforcing on electron number density, shown as the $\log _{10}$ of the ratio between the forced and control runs. The lowchange "stripe" seen at $60-80 \mathrm{~km}$ in the electron number densities and starting at $\sim 11.5$ UT is due to occurrence of sunrise in the model. As previously described (Rodger et al., 2002), WEP produces strong, albeit rather short-lived increases in electron number density in the altitude range from $\sim 75-80 \mathrm{~km}$, and a rather long-lived increase of about $0.5-1$ order of magnitude over a wider altitude range which persists throughout the forcing period. The effect of the WEP ionisation increases on the neutral atmosphere is shown in the lower panels of Fig. 7, presenting the relative change in $\mathrm{NO}_{\mathrm{x}}$ (middle panel) and $\mathrm{O}_{\mathrm{x}}$ (lower panel). The $\mathrm{NO}_{\mathrm{x}}, \mathrm{HO}_{\mathrm{x}}$ and $\mathrm{O}_{\mathrm{x}}$ relative changes are plotted as $\log _{10}(([\mathrm{WEP}$ altered][Background])/[Background]) for the increased levels seen in the $\mathrm{NO}_{\mathrm{x}}$ and $\mathrm{HO}_{\mathrm{x}}$ calculations and $\log _{10}$ ([Background][WEP altered])/[Background]) for the $\mathrm{O}_{\mathrm{x}}$ decreases. In contrast to the electron number density, Fig. 7 indicates that the neutral atmospheric changes driven by WEP are very small. The increase in $\mathrm{NO}_{\mathrm{x}}$ is very low, at most $\sim 0.1 \%$ at $80 \mathrm{~km}$ by the end of the nighttime period. The $\mathrm{HO}_{\mathrm{x}}$ concentration increase is similar, but over a wider range of altitudes $(\sim 70$ $80 \mathrm{~km}$ ). Together the $\mathrm{NO}_{\mathrm{x}}$ and $\mathrm{HO}_{\mathrm{x}}$ enhancements produces an insignificant effect on $\mathrm{O}_{\mathrm{x}}$ as seen in the lower panel, with 

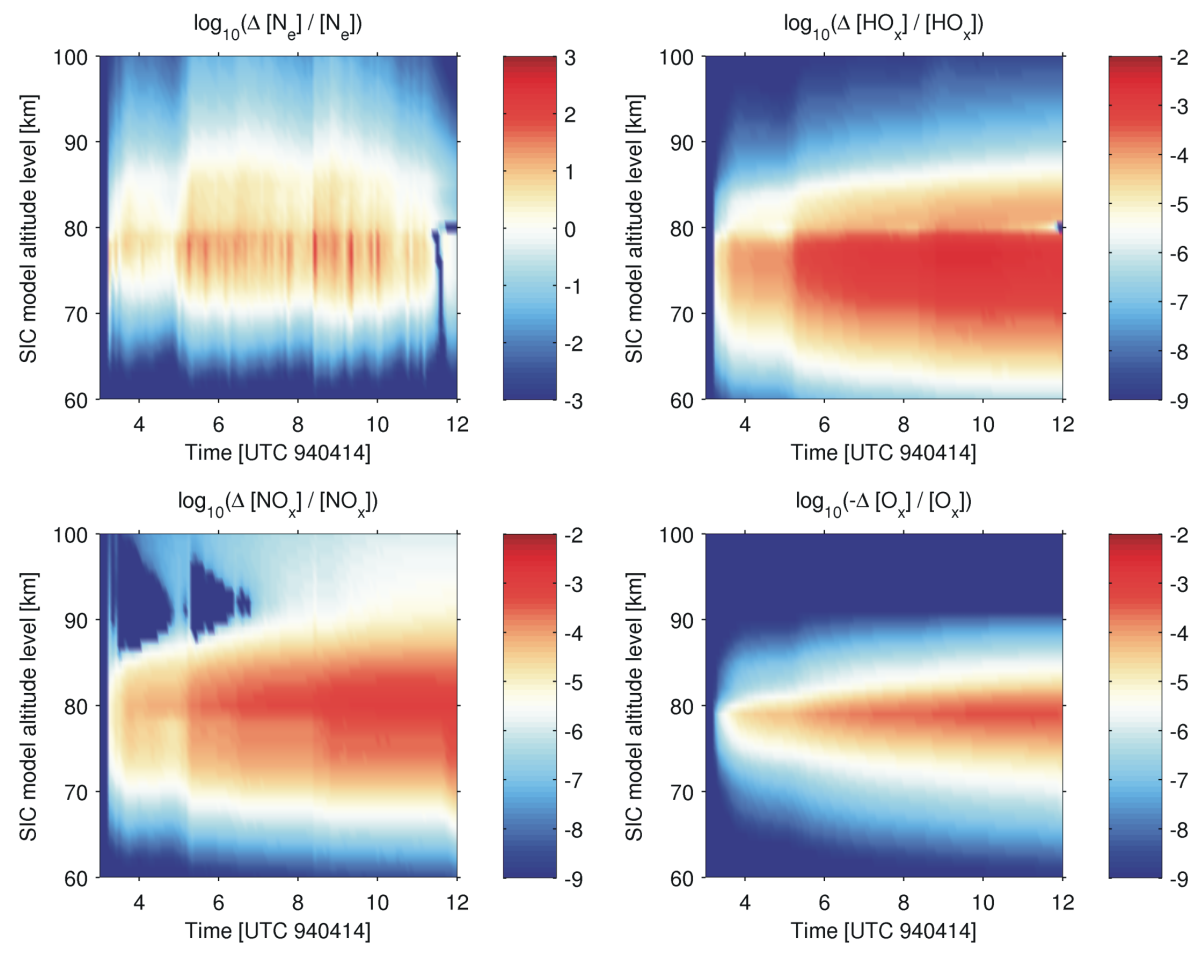

Fig. 7. Effect of the repeated and relatively intense WEP activity shown in Fig. 6, simulated by the SIC atmospheric chemistry model. The panels show the relative change in electron number density, $\mathrm{NO}_{\mathrm{x}}, \mathrm{HO}_{\mathrm{x}}$, and $\mathrm{O}_{\mathrm{x}}$.

a less than $0.1 \%$ decrease at $78 \mathrm{~km}$ at the end of the modelled period. As the nighttime increases in $\mathrm{NO}_{\mathrm{x}}$ will be destroyed through photolysis after sunrise, it is clear that the WEP rate determined from the Trimpi observations will not have a significant effect on the neutral atmosphere in contrast with the ionosphere. The principal reason for this is the short timescale of the WEP bursts. In order to produce significant chemical effects through changes in $\mathrm{NO}_{\mathrm{x}}$, which in our altitudes are due to the dissociation of $\mathrm{N}_{2}$ and proportional to the ionisation rate, long lasting increases in the ionisation rates are required, as occurs during solar proton events (e.g. Seppälä et al., 2004). Significant electron density changes at $80 \mathrm{~km}$ during solar proton events are principally due to the creation of $\mathrm{O}_{2}^{+}$and the ionisation of the background NO. Both the $\mathrm{NO}^{+}$and $\mathrm{O}_{2}^{+}$ions are short-lived.

We noted above that the true WEP rate should be significantly higher than that indicated by Trimpi observations, as the large population of lightning with return stroke peak currents less than $46 \mathrm{kA}$ will produce WEP which will not lead to a measurable Trimpi. Based on the Popolansky peak current probability distribution, the true WEP rate should be $\sim 3.6$ times higher than the Trimpi rate. In order to simulate this, we have randomly generated WEP times and return stroke peak currents such that the extra WEP events are below the Trimpi threshold and such that the WEP rate will be 3.6 times higher that the Trimpi rate observed at that time. While this situation is more likely to represent the true sit- uation, the difference between Fig. 7 and calculations under these conditions is very slight, and almost impossible to detect in the relative plots. We conclude that the Trimpi rate provides a reasonable indication of the WEP rate which will lead to significant electron density changes and neutral chemistry effects, even though these more energetic WEP bursts represent only $\sim 20 \%$ of those occurring.

We have also used the SIC model to consider the ionospheric atmospheric impacts of WEP during normal conditions, where larger return stroke lightning currents are required to produce the same WEP energy flux. While the change to less disturbed conditions slightly alters the range of altitudes affected, it does not substantially alter Figs. 6 or 7 , or lead to a change in the conclusions drawn from these figures.

\section{Discussion}

Throughout our study, we have assumed that WEP bursts are produced by whistlers propagating through the plasmasphere inside whistler ducts, and thus that the cyclotron resonance will be primarily taking place with counter-streaming energetic electrons through the $m=1$ resonance mode. Some studies have suggested that the dominant source of whistlerdriven precipitation will come from non-ducted whistlers, and significant theoretical work has been undertaken to describe the nature of WEP produced by this oblique whistler 
propagation (e.g. Bortnik et al., 2006). Such WEP events would have harder spectra at higher latitudes than in the ducted case, and would also produce longer lasting precipitation bursts than the $0.2 \mathrm{~s}$ events we use here (following S81-1 satellite experimental measurements). There is experimental evidence to support the occurrence of non-ducted whistlerdriver WEP. The size of the ionospheric region affected by each WEP burst is spatially large (at least $600 \mathrm{~km} \times 1500 \mathrm{~km}$ ) (Clilverd et al., 2002), considerably larger than the observed dimensions of whistler ducts (e.g. Angerami, 1970). In addition, a case study of subionospheric observations of WEP made at multiple locations showed increasing time delays with $L$, interpreted as being caused by the drift in $L$ of non-ducted whistlers (Johnson et al., 1999). However, both these sets of observations are also consistent with the quasitrapped whistler propagation theory in which ducted energy spreads at the magnetic equator (Strangeways, 1999), resulting in whistler-mode signals which have leaked outside their whistler duct still contributing to the horizontal lateral extent of WEP. Such leakage would give a significantly larger precipitation footprint than the actual dimensions of the whistler duct, and due to lengthening geomagnetic field-lines, increasing time delays with $L$. Thus the relative importance of ducted or non-ducted whistlers as sources of WEP has been unclear.

Most recently, the propagation of VLF communication transmitter signals into the plasmasphere has been examined by plasma wave instruments onboard the CRRES and DEMETER satellites in order to determine if non-ducted waves are significant in radiation belt loss processes (Clilverd et al., $2007^{1}$ ). The combination of the spacecraft provides observations in the regions where strong transmitter signals are observed in the ionosphere directly above the transmitter, in the magnetosphere near where the signals cross the geomagnetic equator, and also in the ionospheric region geomagnetically conjugate to the transmitter. The study found that non-ducted propagation paths are only significant for low Lshells $(L<1.5)$. At higher $L$-shells the waves become highly ducted in the plasmasphere, with no observed evidence for any non-ducted wave power in the plasmasphere in the range $2<L<3$. As our calculations are guided by the S81-1 satellite observations of WEP, and thus limiting our area of interest to $L=1.9-3.5$, the recent Clilverd et al. (2007) ${ }^{1}$ study suggests that we do not need to include the effects of nonducted whistlers in our calculations. However, we note that modifications will be required to our calculations in the event that non-ducted whistlers are found to be significant for $L>2$.

\footnotetext{
${ }^{1}$ Clilverd, M. A., Rodger, C. J., Gamble, R. J., Meredith, N. P., Parrot, M., Berthelier, J.-J., and Thomson, N. R.: Man-made transmitter signals observed from satellite: ducted or nonducted?, J. Geophys. Res., in review, 2007.
}

\section{Summary}

Lightning-generated whistlers lead to coupling between the troposphere, the Van Allen radiation belts and the lowerionosphere through Whistler-induced electron precipitation. Whistlers can resonate with radiation belt electrons through cyclotron interactions, leading to precipitation into the atmosphere from the newly populated bounce-loss cone. Previous studies have identified Whistler-induced Electron Precipitation (WEP) as a significant inner radiation belt loss process, and have described the how the WEP energy spectra varies with geomagnetic latitude, and the WEP flux magnitude varies with latitude and longitude. We have built on this earlier work to consider the relative significance of WEP to the lower ionosphere and atmosphere.

In order to determine the importance of WEP as an ionisation source in the lower ionosphere, WEP-driven ionisation rates were contrasted with the well known rates caused by galactic cosmic radiation and solar photoionisation. WEPdriven ionisation rates vary both with altitude and location on the Earth. Satellite observations of WEP indicate there is essentially no activity outside of $L=1.9-3.5$, leading to bands of magnetic latitudes in which WEP ionisation rates may be non-zero. The variation in global mean lightning activity drives a very strong variation in WEP-driven ionisation rates across the globe, spatially varying from a low of zero above some ocean regions to highs above North America. As the energy spectrum of WEP bursts softens with increasing magnetic latitude, the peak ionisation rates are located at larger $L$-shells and higher altitudes, where high-fluxes of low energy electrons will be deposited. Below $55 \mathrm{~km}$ there is essentially no WEP-driven ionisation produced as the energy spectrum is too soft, and significant penetration does not occur to these altitudes. Below $60 \mathrm{~km}$ the mean WEP-driven ionisation rate is less than $2 \times 10^{-4}$ electrons $\mathrm{cm}^{-3} \mathrm{~s}^{-1}$ for all locations, insignificant when contrasted with other ionisation sources occurring at these altitudes of which GCR is dominant.

Galactic cosmic radiation (GCR) penetrate deeply into the atmosphere, leading to relatively high ionisation rates at comparatively low altitudes, where the GCR dominates over other ionisation sources. GCR is more significant than WEP at low altitudes for all locations, up to $68 \mathrm{~km}$ altitude where WEP starts to dominate over North America and Central Europe. Between 75 and $80 \mathrm{~km}$ altitude WEP becomes more significant than GCR for the majority of spatial locations at which WEP deposits energy. For most of the mesosphere, the dominant source of ionisation during daytime is solar Lyman-alpha. While WEP drives a higher ionisation rate than daytime solar photoionisation in some parts of the Earth at altitudes lower than $63 \mathrm{~km}$, at those altitudes the GCRdriven ionisation rate is completely dominant. WEP is never a significant source of ionisation in the lower ionosphere during the day. During nighttime scattering from the geocorona leads to significantly reduced, but non-zero solar Lyman- $\alpha$ 
fluxes, and hence nighttime solar photoionisation. The size of the regions in which WEP is the most important nighttime ionisation source peaks at $\sim 80 \mathrm{~km}$, depending on the relative contributions of WEP and nighttime solar Lyman- $\alpha$.

Previous studies have also shown that particle precipitation can lead to large-scale changes in the chemical makeup of the neutral atmosphere. Precipitation-driven increases in ionisation rates enhance minor chemical species that play key roles in the ozone balance of the middle atmosphere. We used the Sodankylä Ion Chemistry (SIC) model to consider the atmospheric consequences of WEP, by focusing on a case-study period where relatively intense WEP activity was observed in Antarctica. The SIC-modelling found that WEP produces strong but short-lived increases in electron number density over $\sim 75-80 \mathrm{~km}$, but accompanied by a rather long-lived increase of about $0.5-1$ orders of magnitude over a wider altitude range, persisting throughout the forcing period, confirming earlier studies into this case-study period. The electron densities modelled by SIC also show that only WEP bursts large enough to produce an observable Trimpi perturbation in subionospheric propagation need to be included in the modelling, as the much larger population $(80 \%)$ of weaker bursts are insignificant.

In contrast to the electron number density, the SIC modelling indicated that the neutral atmospheric changes driven by WEP are very small, with very low increases in $\mathrm{NO}_{\mathrm{x}}$ and $\mathrm{HO}_{\mathrm{x}}$, and insignificant effects on $\mathrm{O}_{\mathrm{x}}$. The principal reason for this is the short timescale of the WEP bursts.

Thus, WEP is a significant energy input into some parts of the lower ionosphere, depending on the latitude/longitude and altitude, but does not play a significant role in the chemical makeup of the mesosphere.

Acknowledgements. C. J. Rodger would like to thank H. Wreford of Cromwell for her support. This research was partially supported by the New Zealand Marsden Research Fund contract 02UOO-106. CFE acknowledges funding through the European Community's Human Potential Programme under contract HPRN-CT2002-00216, Coupling of Atmospheric Layers.

Topical Editor U.-P. Hoppe thanks three anonymous referees for their help in evaluating this paper.

\section{References}

Abel, B. and Thorne, R. M.: Electron scattering loss in earth's inner magnetosphere -2 . Sensitivity to model parameters, J. Geophys. Res., 103, 2397-2407, 1998.

Angerami, J. J.: Whistler duct properties deduced from VLF observations made with the OGO 3 satellite near the magnetic equator, J. Geophys. Res., 75, 6115-6135, 1970.

Banks, P. M. and Kockarts, G.: Aeronomy, vol. B, chap. 15, Academic Press, 1973.

Bortnik, J., Inan, U. S., and Bell, T. F.: Temporal signatures of radiation belt electron precipitation induced by lightning generated MR whistler waves. Part I: Methodology, J. Geophys. Res., 111, A02204, doi:10.1029/2005JA011182, 2006.
Brasseur, G. and Solomon, S.: Aeronomy of the Middle Atmosphere, second ed., D. Reidel Publishing Company, Dordrecht, 1986.

Carpenter, D. L. and LaBelle, J. W.: A study of whistlers correlated with bursts of electron precipitation near $\mathrm{L}=2$, J. Geophys. Res., 87, 4427-4434, 1982.

Carpenter, D. L. and Orville, R. E.: The excitation of active whistler mode signal paths in the magnetosphere by lightning -2 case studies, J. Geophys. Res., 94, 8886-8894, 1989.

Chabrillat, S., Kockarts, G., Fonteyn, D., and Brasseur, G.: Impact of molecular diffusion on the $\mathrm{CO}_{2}$ distribution and the temperature in the mesosphere, Geophys. Res. Lett., 29, 1-4, 2002.

Christian, H. J., Blakeslee, R. J., Boccippio, D. J., Boeck, W. L., Buechler, D. E., Driscoll, K. T., Goodman, S. J., Hall, J. M., Koshak, W. J., Mach, D. M., and Stewart, M. F.: Global frequency and distribution of lightning as observed from space by the Optical Transient Detector, J. Geophys. Res., 108(D1), 4005, doi:10.1029/2002JD002347, 2003.

Clilverd, M. A., Nunn, D., Lev-Tov, S. J., Inan, U. S., Dowden, R. L., Rodger, C. J., and Smith, A. J.: Determining the size of lightning-induced electron precipitation patches, J. Geophys. Res., 107(A8), 1168, doi:10.1029/2001JA000301, 2002.

Clilverd, M. A., Rodger, C. J., and Nunn, D.: Radiation belt electron precipitation fluxes associated with lightning, J. Geophys. Res., 109(12), A12208, doi:10.1029/2004JA010644, 2004.

Dowden, R. L., Adams, C. D. D., Brundell, J., and Dowden, P. E.: Rapid onset, rapid decay (RORD), phase and amplitude perturbations of VLF subionospheric transmissions, J. Atmos. Terr. Phys., 56, 1513-1527, 1994.

Dungey, J. W.: Loss of Van Allen electrons due to whistlers, Planet. Space Sci., 11, 591-595, 1963.

Friedel, R. H. W. and Hughes, A. R. W.: Trimpi events on low latitude paths - an investigation of gyroresonance interactions at low L-values, J. Atmos. Terr. Phys., 54, 1375-1386, 1992.

Heaps, M. G.: Parametrization of the cosmic ray ion-pair production rate above $18 \mathrm{~km}$, Planet. Space Sci., 26(6), 513-517, 1978.

Hedin, A. E.: Extension of the MSIS thermospheric model into the middle and lower atmosphere, J. Geophys. Res., 96, 1159-1172, 1991.

Inan, U. S., Walt, M., Voss, H. D., and Imhof, W. L.: Energy spectra and pitch angle distributions of lightning-induced electron precipitation: Analysis of an event observed on the S81-1 (SEEP) satellite, J. Geophys. Res., 94, 1379-1401, 1989.

Inan U. S., Bell, T. F., Bortnik, J., and Albert, J. M.: Controlled precipitation of radiation belt electrons, J. Geophys. Res., 108, 1186, doi:10.1029/2002JA009580, 2003.

Johnson, M. P., Inan, U. S., and Lauben, D. S.: Subionospheric VLF signatures of oblique (nonducted) whistler-induced precipitation, Geophys. Res. Lett., 26, 3569-3572, 1999.

Kennel, C. F. and Petschek, H. E.: Limit on stably trapped particle fluxes, J. Geophys. Res., 71(1), 1-27, 1966.

Kazil, J., Kopp, E., Chabrillat, S., and Bishop, J.: The University of Bern Atmospheric Ion Model: Time-dependent modelling of the ions in the mesosphere and lower thermosphere, J. Geophys. res., 108(D14), 4432, doi:10.1029/2002JD003024, 2003.

Mackerras, D., Darveniza, M., Orville, R. E., Williams, E. R., and Goodman, S. J.: Global lightning: Total, cloud and ground flash estimates, J. Geophys. Res., 103, 19791-19809, 1998.

Meredith, N. P., Horne, R. B., Iles, R. H. A., Thorne, R. 
M., Heynderickx, D., and Anderson, R. R.: Outer zone relativistic electron acceleration associated with substormenhanced whistler mode chorus, J. Geophys. Res., 108, 1016, doi:10.1029/2001JA900146, 2002.

Menk, F. W., Orr, D., Clilverd, M. A., Smith, A. J., Waters, C. L., Milling, D. K., and Fraser, B. J.: Monitoring spatial and temporal variations in the dayside plasmasphere using geomagnetic field line resonances, J. Geophys. Res., 104(A9), 19955-19969, 1999.

Pasko, V. P. and Inan, U. S.: Recovery signatures of lightningassociated VLF perturbations as a measure of the lower ionosphere, J. Geophys. Res., 99, 17 523-17 537, 1994.

Picone, J. M., Hedin, A. E., Drob, D. P., and Aikin, A. C.: NRLMSISE-00 empirical model of the atmosphere: Statistical comparisons and scientific issues, J. Geophys. Res., 107(A12), 1468, doi:10.1029/2002JA009430, 2002.

Popolansky, F.: Frequency distribution of amplitudes of lightning currents, Electra., 22, 139-146, 1972.

Rees, M. H.: Physics and chemistry of the upper atmosphere, Cambridge University Press, Cambridge, 1989.

Rodger, C. J.: Subionospheric VLF perturbations associated with lightning discharges, J. Atmos. Sol. Terr. Phys., 65, 591-606, 2003.

Rodger, C. J. and Clilverd, M. A.: Inner radiation belt electron lifetimes due to whistler-induced electron precipitation (WEP) driven losses, Geophys. Res. Lett., 29(19), 1924, doi:10.1029/2002GL015795, 2002.

Rodger, C. J., Clilverd, M. A., and Dowden, R. L.: D region reflection height modification by whistler-induced electron precipitation, J. Geophys. Res., 107(7), A7, doi:10.1029/2001JA000311, 2002.

Rodger, C. J., Clilverd, M. A., and McCormick, R. J.: Significance of lightning generated whistlers to inner radiation belt electron lifetimes, J. Geophys. Res., 108(12), 1462, doi:10.1029/2003JA009906, 2003.

Rodger, C. J., McCormick, R. J., and Clilverd, M. A.: Testing the importance of precipitation loss mechanisms in the inner radiation belt, Geophys. Res. Lett., 31(10), L10803, doi:10.1029/2004GL019501, 2004a.

Rodger, C. J., Nunn, D., and Clilverd, M. A.: Investigating radiation belt losses though numerical modelling of precipitating fluxes, Ann. Geophys., 22, 3657-3667, 2004b.

Rodger, C. J., Clilverd, M. A., Thomson, N. R., Nunn, D., and Lichtenberger, J.: Global prediction of inner radiation belt energy deposition caused by lightning, Ann. Geophys., 23, 34193430, 2005, http://www.ann-geophys.net/23/3419/2005/.

Rodger, C. J., Clilverd, M. A., Ulich, Th., Verronen, P. T., Turunen, E., and Thomson, N. R.: The atmospheric implications of Radiation Belt Remediation, Ann. Geophys., 24, 2025-2041, 2006, http://www.ann-geophys.net/24/2025/2006/.

Rycroft, M. J.: Enhanced energetic electron intensities at $100 \mathrm{~km}$ altitude and a whistler propagating through the plasmasphere, Planet. Space Sci., 21, 239-251, 1973.
Seppälä, A., Verronen, P. T., Kyrölä, E., Hassinen, S., Backman, L., Hauchecorne, A., Bertaux, J. L., and Fussen, D.: Solar proton events of October-November 2003: Ozone depletion in the Northern hemisphere polar winter as seen by GOMOS/Envisat, Geophys. Res. Lett., 31(19), L19107, doi:10.1029/2004GL021042, 2004.

Shimazaki, T.: Minor Constituents in the Middle Atmosphere (Developments in Earth and Planetary Physics, No 6), D. Reidel Publishing Co., Dordrecht, Netherlands, 1984.

Storey, L. R. O.: An investigation of whistling atmospherics, Phil. Trans. Roy. Soc. (London), 246, 113-117, 1953.

Strangeways, H. J.: Lightning induced enhancements of D region ionisation and whistler ducts, J. Atmos. Sol. Terr. Phys., 61, 1067-1080, 1999.

Teague, M. and Vette, J.: The inner zone electron model AE-5, National Space Science Data Center, Rep. NSSDC 72-10, Greenbelt, Md., 1972.

Thomas, L. and Bowman, M. R.: A study of pre-sunrise changes in negative ions and electrons in the D-region, Ann. Geophys., 4, 219-227, 1986, http://www.ann-geophys.net/4/219/1986/.

Thomas, L. and Bowman, M. R.: Model studies of the D-region negative-ion composition during day-time and night-time, J. Atmos. Terr. Phys., 47(6), 547-566, 1985.

Tobiska, W. K., Woods, T., Eparvier, F., Viereck, R., Floyd, L. D. B., Rottman, G., and White, O. R.: The SOLAR2000 empirical solar irradiance model and forecast tool, J. Atmos. Terr. Phys., 62, 1233-1250, 2000.

Tsurutani, B. T. and Lakhina, G. S.: Some basic concepts of waveparticle interactions in collisionless plasmas, Rev. Geophys., 35(4), 491-501, 1997.

Turunen, E., Matveinen, H., Tolvanen, J., and Ranta, H.: D-region ion chemistry model, in: STEP Handbook of Ionospheric Models, edited by: Schunk, R. W., pp. 1-25, SCOSTEP Secretariat, Boulder, Colorado, USA, 1996.

Verronen, P. T., Turunen, E., Ulich, Th., and Kyrölä, E.: Modelling the effects of the October 1989 solar proton event on mesospheric odd nitrogen using a detailed ion and neutral chemistry model, Ann. Geophys., 20, 1967-1976, 2002, http://www.ann-geophys.net/20/1967/2002/.

Verronen, P. T., Seppälä, A., Clilverd, M. A., Rodger, C. J.., Kyrölä, E., Enell, C-F., Ulich, Th., and Turunen, E.: Diurnal variation of ozone depletion during the October-November 2003 solar proton event, J. Geophys. Res., 110(A9), A09S32, doi:10.1029/2004JA010932, 2005.

Vampola, A. L.: Outer Zone Energetic Electron Environment Update, European Space Agency P.O. \#151351, 1996.

Voss, H. D., Walt, M., Imhof, W. L., Mobilia, J., and Inan, U. S.: Satellite observations of lightning-induced electron precipitation, J. Geophys. Res., 103, 11 725-11 744, 1998. 